\title{
PRÉ-NATAL MASCULINO: UM RELATO DE EXPERIÊNCIA NO CONTEXTO DA EDUCAÇÃO EM SAÚDE
}

Layres Canuta Cardoso Climaco ${ }^{1}$ Alba Benemérita Alves Vilela ${ }^{1}$ Eduardo Nagib Boery ${ }^{1}$

Sérgio Donha Yarid ${ }^{1}$

\author{
https://orcid.org/0000-0001-8666-5203 \\ https://orcid.org/0000-0002-1187-0437 \\ https://orcid.org/0000-0001-7624-4405 \\ https://orcid.org/0000-0003-0232-4212
}

Objetivo: relatar a vivência de uma enfermeira com o pré-natal masculino na perspectiva de educação em saúde. Método: trata-se de um relato de experiência, que ocorreu por meio do estágio da pós-graduação em Enfermagem e Obstetrícia, de junho a julho de 2018, em uma Unidade Básica de Saúde. Resultados: a vivência permitiu refletir sobre a atuação do profissional de Enfermagem Obstétrica e a construção de saberes voltados para a saúde do homem. A realização de atividade na sala de espera não teve efeito desejado, mas, por outro lado, a consulta do pré-natal se mostrou como um espaço de reflexão e mudança de hábitos a partir da troca de experiências. Considerações finais: o pré-natal masculino vem se mostrando como importante estratégia de incentivo à participação do homem no serviço de saúde, e a educação em saúde se configura como uma estratégia de fortalecimento da atuação do enfermeiro obstetra.

Descritores: Cuidado Pré-Natal; Saúde do Homem; Educação em Saúde; Cuidados de Enfermagem.

\section{MALE PRE-CHRISTMAS: A REPORT OF EXPERIENCE IN THE CONTEXT OF HEALTH EDUCATION}

Objective: to report the experience of a nurse with male prenatal care in the perspective of health education. Method: This is an experience report, which was carried out through the Nursing and Obstetrics post-graduation, from June to July, 2018, in a Basic Health Unit. Results: the experience allowed us to reflect on the performance of the professional obstetric nursing and the construction of family-oriented knowledge, expanding the field of care and knowledge. The activities in waiting room was not effective, but, on the other hand, the prenatal consultation proved to be a space for reflection and change of habits based on the exchange of experience. Final considerations: male prenatal care has been shown to be an important strategy to encourage the participation of men in the health service, and health education is an artifice to strengthen the performance of obstetrician nurses.

Descriptors: Prenatal Care; Men's Health; Health Education; Nursing Care.

\section{PRÉ-NATAL MASCULINO: UN RELATO DE EXPERIENCIA EN EL CONTEXTO DE LA EDUCACIÓN EN SALUD}

Objetivo: relatar la vivencia de una enfermera con el prenatal masculino en la perspectiva de educación en salud. Método: se trata de un relato de experiencia, que ocurrió por medio de la etapa del postgrado en Enfermería y Obstetricia, de junio a julio de 2018, en una Unidad Básica de Salud. Resultados: la vivencia permitió reflexionar sobre la actuación del proceso, profesional de Enfermería Obstétrica y la construcción de saberes orientados a la familia, ampliando el campo de cuidado y conocimiento. La realización de actividades en la sala de espera no fue efectiva, pero, por otro lado, la consulta del prenatal se mostró como un espacio de reflexión y cambio de hábitos a partir del intercambio de experiencia. Consideraciones finales: el prenatal masculino se viene mostrando como importante estrategia de incentivo a la participación del hombre en el servicio de salud, y la educación en salud se configura como un artificio de fortalecimiento de la actuación del enfermero obstetra.

Descriptores: Cuidado Pre-Natal; Salud del hombre; Educación en Salud; Cuidados de Enfermería.

1Programa de Pós-graduação em Enfermagem e Saúde (PPGES) da Universidade do Sudoeste da Bahia (UESB). Autor Correspondente: Layres Canuta Cardoso Climaco Email:laycanuta@gmail.com

Recebido: 01/03/2019 Aceito: 20/11/2019 


\section{INTRODUÇÃO}

A saúde do homem vem sendo discutida no meio científico devido à grande divulgação dos dados epidemiológicos e à criação de políticas públicas voltadas para essa população(1). Nesse contexto, o pré-natal masculino é uma estratégia que visa à valorização dos modelos masculinos por meio do acoIhimento, capacidade de escuta qualificada e possibilidade de inserção dos homens nos serviços de saúde ${ }^{(2)}$.

O pré-natal masculino compreende a gestação como um fenômeno que vai além da concepção, por isso, necessita de ações que envolvam o homem em todo o planejamento familiar até o pós-parto(3). Para o autor, a participação do homem no pré-natal amplia o leque de cuidados à saúde, tanto para mulher, como para a sua própria saúde, em especial, no que diz respeito às Infecções Sexualmente Transmissiveis (IST).

Ao considerar a diversidade de agravos que acometem os homens e que poucos frequentam os serviços de saúde, - Ministério da Saúde (MS), por meio da Portaria GM/MS no 1944, criou a Política Nacional de Atenção Integral à Saúde do Homem (PNAISH), que tem como objetivo promover ações de saúde que contribuam expressivamente para a compreensão da realidade do universo masculino. Essa politica é composta por seis eixos, dentre eles, a paternidade e o cuidado, que visam sensibilizar os gestores, profissionais e a sociedade para vislumbrar os benefícios da paternidade ativa, a fim de prevenir os riscos à sua saúde e a do concepto, bem como a atuação do pai no pré-natal, parto, pós-parto e desenvolvimento infantil(4.5).

No cenário atual do pré-natal, os serviços de saúde desenvolvem atividades exclusivamente voltadas para a gestante, o que torna difícil a participação do parceiro no processo gravídico(b). Isso é um desafio para os profissionais, sendo necessário mudar a configuração do serviço e a perspectiva de cuidado que busque envolver as gestantes e os seus parceiros.

Com isso, é importante destacar que o pré-natal é um momento propício para enfatizar o poder da educação em saúde, que é compreendida como uma ação prévia de promoção da saúde, que atua de forma participativa e reflexiva, possibilitando, às pessoas, tomarem decisões conscientes que influenciam o processo saúde/doença ${ }^{(7)}$. Acredita-se ainda que, para se promover a educação em saúde, se deve enfatizar a educação popular em saúde, a qual envolve os saberes prévios da população, atrelados ao conhecimento científico ofertado(8).

Há uma necessidade de ampliar as ações voltadas para a saúde do homem na atenção básica, sendo o enfermeiro um elemento fundamental para a transformação deste cenário, ao prestar uma assistência empática, acolhimento atrativo e humanizado, de forma holística e integral(9). Assim, para a Enfermagem, no pré-natal, existem diversos espaços para atuar na perspectiva da educação em saúde, tais como: sala de espera, assistência de Enfermagem, consultas e grupos de gestantes.

Nessa perspectiva, este estudo tem como objetivo: relatar a vivência de uma enfermeira com o pré-natal do parceiro na Unidade Básica de Saúde (UBS) na perspectiva de educação em saúde.

\section{MÉTODO}

\section{Tipo de estudo}

Estudo descritivo, do tipo relato de experiência, com abordagem qualitativa, que consistiu em descrever a vivência de uma enfermeira com a educação em saúde no pré-natal masculino.

\section{Local do estudo}

Realizado no serviço de pré-natal da Unidade Básica de Saúde Almerinda Lomanto, localizada em Jequié, Bahia, Brasil, que ocorreu por meio de estágio prático da Pós-Graduação em Enfermagem Obstétrica. A experiência ocorreu de junho a julho de 2018, com carga horária de 40 horas, distribuidas de segunda a quinta-feira.

\section{Participantes da pesquisa}

Enfermeira pós-graduanda e docente.

\section{Coleta de dados}

Foi por meio de um diário de campo que constava o planejamento das atividades propostas pela docente e desenvolvimento tanto da sala de espera e como da consulta. Assim, esse diário foi registrado anotações diariamente sobre o estágio tais como: data; horário; duração; local da observação; tema abordado na sala de espera; descrição do espaço físico; recursos didáticos; estratégia metodológica; quantidade de homens, gestantes, acompanhantes, profissionais; aparência fisica; expressões faciais; gestos; materiais utilizados para consulta (impressos, caderneta, testes rápidos); descrição da consulta; e a observação dos acontecimentos particulares (sentimentos, problemas, ideias, palpites, impressões, facilidades e dificuldades encontradas, resultados alcançados). Além disso, no diário de campo constava as informações sobre as discussões entre os discentes e a docente, particularmente sobre os aspectos que chamou a atenção durante a abordagem da sala de espera; a participação do homem durante o pré-natal; e as impressões sobre o serviço e o acolhimento do homem. 


\section{Procedimentos de análise dos dados qualitativos}

As informações do diário de campo foram transcritas e realizada uma análise compreensiva das informações. No primeiro momento, realizou-se uma leitura do material obtido. Na sequência, através da exploração do material foi realizado um quadro construído por uma das autoras que constava do lado esquerdo as informações do diário e do lado direito as reflexões e especulações sobre o que está emergindo.

\section{Aspectos éticos}

Por se tratar de um relato de experiência não foi necessário a submissão ao Comitê de Ética em Pesquisa. Mesmo tratando-se desse tipo de estudo, todos os princípios éticos foram seguidos, conforme as recomendações nacionais e internacionais de pesquisa, e ainda, vale ressaltar que obteve-se a anuência da instituição para publicação da experiência.

\section{RESULTADOS DA EXPERIÊNCIA}

$\mathrm{Na}$ sala de espera, foram realizadas rodas de conversa com os seguintes temas: aleitamento materno, cuidado com coto umbilical, trabalho de parto e violência obstétrica. Nesse momento, além de abordar a temática do dia, buscaram-se esclarecer mitos e dúvidas sobre o tema escolhido e explorar as vivências de gestações anteriores com o propósito de estimular a reflexão sobre os temas e mudanças de hábitos que não são favoráveis para o pré-natal, parto e puerpério. Com o objetivo de melhorar a abordagem do processo de educação em saúde, a troca de conhecimentos foi realizada de forma atrativa e lúdica, por meio de álbum seriado, mamas e bonecos didáticos, que serviram para estabelecer o vínculo com os usuários.

A consulta do pré-natal foi desenvolvida da seguinte forma: primeiramente, acolheram-se a gestante e o parceiro. $\mathrm{Na}$ sequência, parabenizou-se o futuro papai pela sua participação naquele espaço, reconhecendo o seu protagonismo no contexto do pré-natal. Ao mesmo tempo em que se escutaram as queixas do homem, forneceram-se as devidas orientações referentes à saúde do mesmo, sendo pode também ser responsável pelo seu estado de saúde.

$\mathrm{Na}$ sequência, foram ofertadas outras ações, tais como: verificação dos sinais vitais, abordagem da importância da detecção precoce de doenças, atualização da caderneta de vacina e atividades educativas.

Foi realizado, pela pós-graduanda em Enfermagem Obstétrica, o aconselhamento do pré-teste rápido de hepatites $\mathrm{B}$ e C, anti-HIV e sífilis, após apresentar, para os homens, em cada consulta, separadamente, as principais doenças que podem ocorrer no pré-natal; com sua autorização, fazia-se a coleta de sangue para o procedimento. $\mathrm{Na}$ espera pelos resultados dos testes, esses homens aproveitavam para tirar dúvidas sobre alimentação, sintomas mais frequentes da gestação e anemia falciforme. No exame físico da gestante, os parceiros davam suporte à companheira e se emocionavam com os batimentos cardiofetais (BCF)

$\mathrm{O}$ aconselhamento pré-teste rápido de hepatites $\mathrm{B}$ e $\mathrm{C}$, anti-HIV e sífilis também foi uma estratégia de educação em saúde que se destacou, pois houve a troca de informações entre a enfermeira e o parceiro da gestante sobre IST, principalmente, no que diz respeito aos perigos da sífilis durante a gravidez, desde a má-formação fetal, até mesmo o aborto. Essa ação contribuiu para o controle de doenças, tais como: hepatites B e C, HIV e sifilis.

Outro aspecto importante diz respeito ao atual estado de saúde. Pôde-se perceber que muitos homens não tinham o cartão vacinal, não faziam exames laboratoriais e a procura por cirurgião-dentista acontecia apenas quando tinham uma patologia já instalada. Nesse momento, a educação em saúde foi importante, pois foi possivel alerta-los sobre os cuidados que os mesmos devem ter com a saúde, fazendo com que passassem a refletir e a tomar decisões, tais como: ir à sala de vacina, realizar os testes rápidos no momento da consulta, bem como ir ao odontólogo e realizar exames laboratoriais.

\section{DISCUSSÃO}

\section{A sala de espera no contexto da educação em saúde}

Sabe-se que as rodas de conversa estabelecem uma postura ético-política em relação à produção do conhecimento e, por serem uma estratégia de transformação social, partindo da reflexão e negociação entre os profissionais e usuários, tem a intenção de construir novas possibilidades, por meio do perceber, refletir, agir e modificar ${ }^{(10)}$. No entanto, percebe-se que a sala de espera não teve o efeito desejado, visto que o público masculino não estava à vontade naquele ambiente e não interagiu durante o momento da abordagem dos temas escolhidos. Isso reflete a necessidade do profissional desenvolver a capacidade de se comunicar com o público, motivando os homens a participarem das atividades de educação em saúde e a usar o serviço de saúde.

Além disso, alguns autores retratam que a deficiência da sala de espera no serviço é por não oferecer tempo adequado para a escuta ou, até mesmo, para a construção crítica coletiva ${ }^{(11)}$. Estudo ${ }^{(12)}$ aponta que a formação do enfermeiro para atuação no campo da educação em saúde precisa ser revista, pois, muitas vezes, estas ações ofertadas nos serviços de saúde não estão direcionadas para um processo que esteja contemplando a visão de mundo dos sujeitos.

Nesse sentido, a educação em saúde só seria efetiva se os profissionais retomassem alguns princípios, tais como, a 
comunicação, a informação, a educação e a escuta qualificada, para, assim, os profissionais visualizarem novas formas de intervir na realidade de saúde dos usuários $^{(7)}$, ou seja, implementarem novas ações que venham a modificar o universo masculino.

Quanto às temáticas de aleitamento materno, cuidado com coto umbilical, trabalho de parto e violência obstétrica favoreceram as práticas em promoção de saúde e a sensibilização do homem no contexto gravídico, uma vez que se buscou refletir sobre a inclusão do pai no cuidado em saúde durante a gravidez até o puerpério. Ao analisar a vivência, percebe-se que as ações antes ofertadas no serviço de saúde estavam voltadas para a gestante e que não existem ações integrais e universais, conforme os principios do SUS, que envolvam o homem como participante ativo no seu processo saúde/doença.

No entanto, essa realidade já deveria ser diferente por já existir uma política voltada para público masculino. Ao refletir sobre o que versa a PNAISH, constatam-se as fragilidades da Atenção Básica no que diz respeito à assistência do cuidado oferecido aos homens. Isso corrobora a situação encontrada no estudo, em que se abordam as dificuldades para a inserção dos homens na atenção primária ${ }^{(13)}$. Dentre elas, estão a ausência desse público no serviço, o deficit de capacitação dos profissionais sobre a temática, a escassez de conhecimento sobre a PNAISH pelos profissionais e, além disso, existe uma feminização no serviço que interfere no cuidado à saúde do homem(13).

No entanto, fica claro para os profissionais de saúde que é preciso buscar estratégias para tornarem o homem visivel no serviço, sendo necessário romper velhos paradigmas que impedem que o público masculino participe mais ativamente do seu cuidado e reflita sobre a sua situação de saúde ${ }^{(14,15)}$.

\section{Interface: consulta de Enfermagem e Educação em Saúde}

O uso de estratégias de educação em saúde é uma das competências necessárias para o trabalho do enfermeiro em que o profissional, como educador, conduz a transformação dos usuários inseridos no serviço, ampliando a capacidade de compreensão dos mesmos ${ }^{(16)}$. Desse modo, a educação em saúde pode ser usada como uma valiosa estratégia de promoção de saúde e de autocuidado, a partir do momento em que os indivíduos se conscientizem ${ }^{(17)}$

O modelo de aconselhamento se configura como uma prática de saúde que integra a realidade do sujeito e a construção de respostas efetivas em prol da promoção de saú$\mathrm{de}^{(18)}$. Para o autor, existe o modelo de "aconselhamento relação", caracterizado pelo diálogo entre profissional e indivíduo, por se tratar de uma prática horizontal e democrática, incen- tivando também a participação ativa do parceiro.

Essa participação ativa do parceiro no pré-natal possibilita a transformação de atitudes e habilidades relacionadas ao processo gravídico, bem como a conscientização sobre comportamentos preventivos relacionados às IST. Tal relação foi comprovada em um estudo que aborda a inclusão dos homens nos programas de cuidados de saúde das mulheres grávidas, sendo que essa participação é, de fato, sucesso para a melhoria do seu estado de saúde ${ }^{(19)}$

As reflexões feitas sobre a participação do homem nos espaços de saúde serviram para mudanças de conceitos e perspectivas de cuidado pela profissional durante a consulta do pré-natal. No entanto, por mais que alguns autores abordem o processo de ensino-aprendizagem participativo e reflexivo dentro do serviço de saúde como um desafio, esse método permite modificar processos de trabalho nos quais o profissional está inserido(20)

No entanto, a formação profissional em saúde ainda está ligada ao preconceito relacionado à inserção do homem no serviço, prejudicando a consolidação dos preceitos da política nacional voltada para o homem ${ }^{(21)}$. A partir dessa experiência, realizar o pré-natal do parceiro permitiu refletir sobre a atuação profissional da Enfermagem Obstétrica e a construção de saberes voltados para a família e não somente para a gestante, ampliando o campo de cuidado e o conhecimento.

\section{Limitação da experiência}

Traz-se, portanto, como limitação desse estudo a falta de preparo dos profissionais de saúde e a organização do serviço de saúde que impossibilitou ter um espaço exclusivo para o atendimento e acolhimento dos homens na Atenção Primária à Saúde. Dessa forma, é necessário mudar a configuração dos serviços de saúde, envolvendo o homem na oferta de serviços, e que sejam ofertadas ações de educação em saúde mais atrativas, efetivas e voltadas para o público masculino.

\section{Contribuição para a prática}

Para a enfermagem, as ações de educação em saúde permitem: ampliar o acesso do usuário ao serviço de saúde, trabalhar na perspectiva da promoção de saúde e do autocuidado. Além disso, contribuem para a transformação dos usuários, aumentando sua capacidade de compreensão sobre o processo saúde/doença.

\section{CONSIDERAÇÕES FINAIS}

O pré-natal masculino vem se mostrando como importante estratégia de incentivo à participação do homem no serviço de saúde, devendo ser implementado no serviço de Atenção Primária à Saúde, sendo que a educação em saúde se configura como um artifício de fortalecimento da atua- 
ção do profissional que favorece a promoção de saúde e o autocuidado.

Sabe-se que, no contexto do pré-natal, os homens são vistos como pessoas invisíveis e que, na maioria das vezes, os serviços de saúde não têm exercido a função de atender às necessidades de integralidade do cuidado para esse público.

A Enfermagem Obstétrica tem a função de ampliar a visão sobre a participação do homem no pré-natal e contribuir para a construção de indivíduos mais autônomos. Assim, experiência permitiu ampliar o conhecimento sobre a atuação do enfermeiro na perspectiva do cuidado, bem como a inclusão do homem na abordagem no que diz respeito ao pré-natal, parto e puerpério, podendo diminuir os aspectos negativos para a saúde do binômio mãe e filho e, ainda, estender o cuidado ao homem.
É necessário que os enfermeiros, mais especificamente os obstetras, busquem ampliar suas competências voltadas para a saúde do homem, conheçam a PNAISH e modifiquem o seu campo de atuação. Este estudo abre possibilidades para novas pesquisa que envolvam a paternidade ativa. Sugere-se a realização de estudos que avaliem o serviço de pré-natal, na perspectiva do homem, de modo a investigar as facilidades e dificuldades das ações que envolvem a educação em saúde, para que se torne um serviço mais atrativo para os homens.

CONTRIBUIÇÕES DOS AUTORES: Climaco LCC, participou na concepção e delineamento do estudo. VilelaABA, Boery EN, Yarid SD colaboraram na análise e interpretação dos dados, redação e revisão crítica relevante ao conteúdo intelectual do manuscrito e aprovação final da versão a ser publicada.

\section{REFERÊNCIAS}

1.Schwarz E, Gomes R, Couto MT, Erly CM, Carvalho AS, Silva SFC. Política de saúde do homem. Rev Saude Publica. [Internet] 2012 [cited 2018 Aug 18]; 46 Supl 1:108-116. Available from: http://www.scielo.br/scielo.php?script=sci_ arttextEpid=S0034-89102012000700015\&lng=enEnrm=iso

2.Ministério da Saúde (BR). Guia do Pré-Natal do Parceiro para Profissionais de Saúde. Rio de Janeiro (RJ): Ministério da saúde; 2016.

3.Costa SF, Taquette SR. Atenção à gestante adolescente na rede sus - o acolhimento do parceiro no pré-natal. J Nurs UFPE on line. [Internet] 2017 [cited 2018 Aug 21]; 11 (Supl5):2067-2074. Available from: https://periodicos.ufpe. br/revistas/revistaenfermagem/article/viewFile/23360/18984

4.Ministério da saúde (BR). Portaria no 1.944, de 27 de agosto de 2009. Institui no âmbito do Sistema Único de Saúde (SUS), a Política Nacional de Atenção Integral à Saúde do Homem. Brasilia (DF): Diário Oficial da União; 2009.

5.Primo CC, Trevizani CC, Tedesco JC, Leite FMC, Almeida MVS, Lima EFA. Classificação internacional para a prática de enfermagem na assistência pré-natal. Enferm em Foco. [Internet] 2015 [cited 2019 Nov 13]; 6 (1/4): 17-23. Available from: <http://revista.cofen.gov.br/index.php/enfermagem/article/view/571/253>

6.Henz GS, Medeiros CRG, Salvadori M. Paternal inclusion during prenatal care. Rev Enferm Atenção Saúde Online. [Internet] 2017 [cited 2018 Aug 21]; 6(1):52-66. Available from: http://seer.uftm.edu.br/revistaeletronica/index.php/ enfer/article/view/2053/pdf_1

7.Salci MA, Maceno P, Rozza SG, Silva DMGV, Boehs AE, Heidemann ITSB. Health education and its theoretical perspectives: a few reflections. Texto Contexto Enferm. [Internet] 2013 [cited 2018 Aug 24]; 22 (1): 224-230. Available from: http://www.scielo.br/scielo.php?script=sci_arttext\&pid=S0104-07072013000100027\&lng=en\&nrm=iso

8.Falkenberg MB, Mendes TPL; Moraes EP, Souza EM. Educação em saúde e educação na saúde: conceitos e implicações para a saúde coletiva. Cienc Saude coletiva. [Internet] 2014 [cited 2018 Set 24]; 19 (3): 847-852. Available from: http:// www.scielo.br/scielo.php?script=sci_arttextEpid=S141381232014000300847\&lng=enधnrm=iso 
9.Albuquerque GA, Leite MF, Belém JM, Nunes JFC, Oliveira MA, Adam F. The man in primary healthcare: perceptions of nurses about the implications of gender in health. Esc Anna Nery. [Internet] 2014 [cited 2018 Sep 24]; 18 (4): 607-614. Available from: http://www.scielo.br/scielo.php?script=sci_arttext\&pid=S1414-81452014000400607\&lng=enधnrm=iso

10.Sampaio J, Santos GC, Agostini M, Salvador AS. Limits and potentialities of the circles of conversation: analysis of an experience with young people in the backcountry of Pernambuco, Brazil. Interface (Botucatu). [Internet] 2014 [cited 2018 Aug 15]; 18(Supl2):1299-1312. Available from: http://www.scielo.br/scielo.php?script=sci_ arttextEpid=S1414-32832014000601299\&lng=en\&nrm=iso

11. Holanda SM, Castro RCMB, Aquin PS, Pinheiro AKB, Lopes LG, Martins ES. Influência da participação do companheiro no pré-natal: satisfação de primiparas quanto ao apoio no parto. Texto Contexto Enferm. [Internet] 2018 [cited 2019 Oct 12]; 27(2): e3800016. Available from: http://www.scielo.br/scielo.php?script=sci_ arttextEpid=S0104-07072018000200317\&lng=en.

12.Colomé JS, Oliveira DLLC. Educação em saúde: por quem e para quem? A visão de estudantes de graduação em enfermagem. Texto contexto - enferm. [Internet] 2012 [cited 2019 Oct 21]; 21(1):177-184. Available from: http://www. scielo.br/scielo.php?script=sci_arttextEpid=S0104-07072012000100020\&lng=en.

13.Moreira RLSF, Fontes WD, Barboza TM. Dificuldades de inserção do homem na atenção básica a saúde. Esc Anna Nery. [Internet] 2014 [cited 2018 Aug 15]; 18(4): 615-621. Available from: http://www.scielo.br/scielo.php?script=sci_ arttext\&pid=S1414-81452014000400615\&lng=en\&nrm=iso

14.Santana EM, Lima EMM, Bulhões JLF, Monteiro EMLM, Aquino JM. A atenção à saúde do homem: ações e perspectivas dos enfermeiros. Rev Min Enferm. [Internet] 2011[cited 2018 Aug 17]; 15(3): 324-332. Available from: http://www.reme.org.br/artigo/detalhes/41

15.Storino LP, Souza KV, Silva KL. Necessidades de saúde de homens na atenção básica: acolhimento e vínculo como potencializadores da integralidade. Esc Anna Nery. [Internet] 2018 [cited 2018 Aug 17]; 17 (4):638-645. Available from: http://www.scielo.br/scielo.php?script=sci_arttextEpid=S1414-81452013000400638\&lng=ptEnrm=iso

16.Regis CG, Batista NA. The nurse in the area of population health: concepts and competencies. Rev Bras Enferm. [Internet] 2015 [cited 2018 Aug 20]; 68(5):548-54. Available from: http://www.scielo.br/pdf/reben/v68n5/en_00347167-reben-68-05-0830.pdf

17.Richards MJ, Peters M, Sheeder J, Kaul P. Contraception and Adolescent Males: An Opportunity for Providers. Journal of Adolescent Health. [Internet] 2015 [cited 2019 Feb 06]; 58(3): 366 - 368. Available from: https://www. jahonline.org/article/S1054-139X(15)00406-1/fulltext

18.Galindo WCM, Francisco AL, Rios LF. A instrução e a relação como modos de aconselhamento em HIV/Aids. Temas psicol. [Internet] 2013 [cited 2018 Set 11]; 21(3):989-1004. Available from: http://pepsic.bvsalud.org/scielo. php?script=sci_arttextEpid=S1413-389X2013000300014Elng=ptEnrm=iso

19.Karimiankakolakil Z, Mahmoodabad SSM, Kazemi A, Fallahzadeh H. Designing an educational intervention on second-hand smoke in smoker men on the exposure of pregnant wives: a protocol for a randomized controlled trial. Reproductive Health. [Internet] 2019 [cited 2019 Feb 06]; 16:11. Available from: https://reproductive-health-journal. biomedcentral.com/articles/10.1186/s12978-019-0673-1

20.Andrade RS, Caldas LBSN, Falcao MLP, Goes PSA. Processo de trabalho em unidade de saúde da família e a educação permanente. Trab Educ saúde. [Internet] 2016 [cited 2018 Set 11]; 14(2):505-521. Available from: http:// www.scielo.br/scielo.php?script=sci_arttextEpid=S1981-77462016000200505\&lng=en\&nrm=iso

21.Moreira MCN, Gomes R, Ribeiro CR. E agora o homem vem?! Estratégias de atenção à saúde dos homens. Cad Saúde Pública. [Internet] 2016 [cited 2018 Set 11]; 32(4)e00060015. Available from: http://www.scielo.br/scielo. php?script=sci_arttextEpid=S0102-311X2016000400710\&lng=enEnrm=iso 